Collection SFN 8 (2007) 11-22

(C) EDP Sciences, Les Ulis

DOI: $10.1051 / \mathrm{sfn}: 2007003$

\title{
Introduction à la réflectivité de neutrons : potentialités pour les études de surfaces de systèmes magnétiques et en matière molle
}

\author{
G. Fragneto et R. Cubitt \\ Institut Laue-Langevin, 6 rue Jules Horowitz, BP. 156, 38042 Grenoble, France
}

\section{INTRODUCTION}

La réflectivité de la lumière sur une surface est un phénomène bien connu et provoqué par le changement d'indice de réfraction au passage à travers l'interface. Déjà en 1675, Newton avait observé que la couleur d'une couche mince de film de savon illuminé par un faisceau parallèle de lumière blanche, pouvait être utilisépour estimer l'épaisseur de la couche. Les couleurs produites sont le résultat de l'interférence entre la lumière réfléchie en dessous et au-dessus des surfaces de la couche. L'interférence optique est utilisée encore aujourd'hui pour mesurer l'épaisseur de couches sur des surfaces.

Dans les années 20, des expériences menées par Compton ont démontré que la réflexion des rayons $X$ est gouvernée par les mêmes lois de la réflexion de la lumière avec des indices de réfraction dépendant du nombre d'électrons par unité de volume. En 1944, Fermi et Zinn démontraient pour la premiàre fois la réflectivité de neutrons [1]. Encore une fois, les mêmes équations fondamentales de la réflectivité optique sont suivies, mais les indices de réfraction dépendent non seulement du nombre de noyaux des atomes mais aussi de leur pouvoir de diffusion. Une quantité appelée « longueur de diffusion » peut être définie pour chaque noyau et indique son habilité à diffuser les neutrons. L'indice de réfraction d'un matériel dépend de la longueur de diffusion des noyaux qui le composent. Comme dans le cas de la lumière, la réflexion totale peut se produire quand les neutrons traversent deux milieux avec une différence d'indices de réfraction positive. Les indices de réfraction de la plupart de la matière condensée étant inférieurs à ceux de l'air ou du vide, la réflexion totale interne est observée plus couramment que la réflexion totale externe observée avec la lumière. L'angle critique de réflexion totale est tel que la réflectivité de neutrons d'une longueur d'onde donnée à partir d'une interface vaut un aux angles inférieurs et décroît très rapidement aux grands angles. Fermi et Zinn [1] avaient observé la réflexion totale des neutrons thermiques au-dessous de l'angle critique. L'indice de réfraction pour les neutrons dépend de la composition et des longueurs de diffusion du matériel. Par conséquent, la mesure de l'angle critique de réflexion totale a constitué une méthode importante pour déterminer les longueurs de diffusion des noyaux. Pendant longtemps, la réflectivité des neutrons a été utilisé e non seulement pour le transport des neutrons thermiques dans les guides de neutrons, mais aussi pour ces déterminations, même si la présence d'inhomogénéités aux interfaces réduisait la précision des mesures. Ces vingt dernières années, des méthodes plus précises ont été développées pour mesurer les longueurs de diffusion, et la réflectivité des neutrons est devenue une technique puissante pour étudier les inhomogénéités à travers les interfaces mêmes, inhomogénéités dues soit à la composition [2] soit à la magnétisation [3]. Tout comme dans le cas de la lumière, le phénomène d'interférences peut se produire entre les ondes réfléchies en dessus et au-dessous d'une couche, ce qui provoque la formation de franges d'interférences dans le profil de réflectivité. Pour les études aux interfaces, la réflectivité des neutrons offre plusieurs avantages par rapport à des techniques plus traditionnelles ou aux rayons $X$. En effet, à cause des petites longueurs d'onde des neutrons, la technique a une résolution de l'ordre de la fraction de nanomètre. Elle ne provoque pas l'endommagement des échantillons, et peut être utiliséepour étudier des interfaces cachées, qui peuvent très difficilement être étudiées avec d'autres techniques. Elle présente aussi l'avantage de permettre l'utilisation de la substitution isotopique pour atteindre des grands contrastes de densités de longueur de diffusion. Cette méthode sera illustrée dans les nombreux exemples détaillésdans plusieurs chapitres de 
ce livre. L'information sur les profils de concentration, qui sont à l'origine des courbes de réflectivité, est obtenue en affinant les courbes expérimentales à des modèles théoriques. Les inhomogénéités latérales par rapport aux plans des surfaces et interfaces ne peuvent pas être étudiées par réflectivité spéculaire : on a eu recours à des études de diffusion hors spéculaire [4]. Ces dernières ont récemment reçu beaucoup d'attentions et c'est dans cette direction qu'il faut rechercher les potentialités et les développements futurs de la technique.

La réflectivité des neutrons est utilisée pour des études de chimie de surface (tensioactifs, polymères, lipides, protéines et mélanges adsorbés aux interfaces liquide-fluide et solide-fluide), pour étudier le magnétisme aux surfaces (couches ultrafines de Fer, multicouches magnétiques) et la structure de couches solides (couches de Langmuir-Blodgett, couches minces, multicouches, couches de polymères).

Ce manuscrit présente une introduction sur la théorie et les méthodes de mesure de la réflectivité de neutrons avec une attention particulière aux applications dans le domaine de la matière molle.

\section{PRINCIPES THÉORIQUES}

Deux approches de la théorie de la réflectivité de neutrons sont possibles. La première repose sur l'aspect ondulatoire des neutrons et par conséquent une similarité avec le rayonnement lumineux et des théories classiques d'optique [5]. La seconde traite les neutrons comme des particules qui sont décrites par une superposition linéaire d'ondes planes, solutions de l'équation de Schrödinger. La première approche est préférable dans le cas de l'analyse de la réflectivité spéculaire àcause de l'existence d'algorithmes permettant de calculer exactement la réflectivité de n'importe quelle structure normale à la surface ainsi que de solutions exactes et approchées de profils d'interfaces particuliers. En ce qui concerne la réflectivité non-spéculaire, il vaut mieux résoudre l'équation de Schrödinger. Une description plus compréhensible du neutron comme particule et de l'onde réfléchie associée a été proposée par Lekner [6]. L'équation de Schrödinger s'écrit :

$$
-\frac{h^{2}}{8 \pi^{2} m_{n}} \nabla^{2} \Psi+V \Psi=E \Psi
$$

où $h$ est la constante de Planck, $m_{n}$ la masse du neutron, $V$ le potentiel auquel est soumis le neutron et $E$ son énergie. $V$ représente les effets d'interactions entre le neutron et les diffuseurs du milieu qu'il traverse. Dans le cas ou le faisceau diffusé ne remplit pas la condition de Bragg de la structure cristalline traversée, le potentiel $V$ est considéré comme constant, fonction uniquement d'une longueur de diffusion cohérente. Il s'écrit

$$
V=\frac{h^{2}}{2 \pi m_{n}} N_{b}
$$

où $N_{b}$ est la densité de longueur de diffusion définie par :

$$
N_{b}=\sum_{j} b_{j} n_{j}
$$

avec $n_{j}$ le nombre de noyaux par unité de volume et $b_{j}$ la longueur de diffusion du noyau $j$. Cette derniàre est une quantité empirique, connue pour la plupart des noyaux. Comme déjà mentionné dans le cours d'introduction de J. Teixeira (ce livre), cette quantité varie de manière aléatoire à travers la classification périodique des éléments, et varie aussi surtout en fonction des isotopes d'un même élément. La valeur de $b$ de la plupart des matériaux est positive ; ainsi dans un potentiel positif, le neutron va perdre de l'énergie et ainsi correspondre à une longueur d'onde plus grande, contrairement à la lumière qui verra la longueur d'onde diminuer.

Considérons maintenant un faisceau de neutrons approchant une surface, de potentiel en volume $V_{0}$, infiniment profonde (voir Figure 1). 


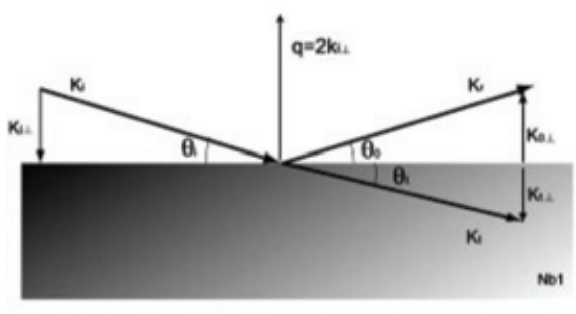

(a)

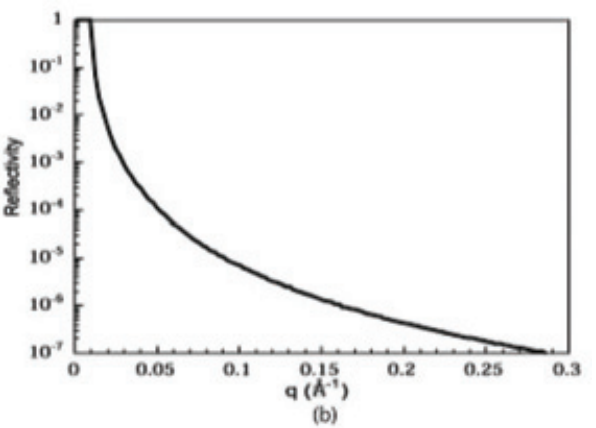

Figure 1. (a) Réflectivité d'un faisceau incident par une surface plane idéale. Ki et Kf sont les vecteurs d'onde incident et diffusé, aux angles $\theta_{i}=\theta_{0}=\theta$ dans le plan d'incidence ; $q$ est le vecteur d'onde de transfert ; $\mathrm{Nb}_{1}$ est la densité de longueur de diffusion du substrat. À droite, le profil de densité de longueur de diffusion en fonction de la profondeur. (b) Simulation de la réflectivité spéculaire de neutrons en fonction de $q$ pour une interface silicium/air $\left(\mathrm{Nb}_{1}=2.0710^{-6} \AA^{-2}\right)$.

S'il n'y a pas de structure le long de la surface, le seul gradient de potentiel, et par conséquent la force, est perpendiculaire à la surface. Seule la composante normale du vecteur d'onde incident, $k_{i}$, sera modifiée par la barrière de potentiel, et c'est la composante normale de l'énergie cinétique $E_{i \perp}$ qui détermine si le neutron est totalement réfléchi ou non.

$$
E_{i \perp}=\left(\frac{\left(h k_{i} \sin \theta_{i}\right)^{2}}{4 \pi m_{n}}\right)
$$

où $\theta_{i}$ est l'angle entre le faisceau incident et la surface.

Lorsque $E_{i \perp}<V_{o}$, il y a réflexion totale. La valeur du vecteur de diffusion critique, $q_{c}$ pour $E_{i \perp}=V_{0}$ est donnée par :

$$
q_{c}=\sqrt{16 \pi N_{b}} \quad \text { et } \quad q=2 k_{i} \sin \theta_{i}
$$

En supposant que la diffusion soit élastique, la condition de conservation des moments implique $\theta_{i}=\theta_{0}$ où $\theta_{0}$ est l'angle du faisceau réfléchi, i.e. la réflexion est spéculaire. Toute réflexion non spéculaire doit être le résultat de gradients de potentiel dans le plan $x y$ de la surface.

Lorsque $E_{i \perp}>V_{0}$, la réflexion n'est plus totale et le neutron peut être soit réfléchi soit transmis à l'intérieur du matériau. Le faisceau transmis $k_{t}$, avec la composante normale de l'énergie cinétique réduite par le potentiel, doit changer de direction, i.e. le faisceau est réfracté. Le changement du vecteur d'onde normal vaut :

$$
k_{t \perp}^{2}=k_{i \perp}^{2}-4 \pi N_{b} \quad \text { ou } \quad q_{t}^{2}=q_{i}^{2}-q_{c}^{2}
$$

On définit alors un indice de réfraction relatif $n$ :

$$
n^{2}=\frac{k_{t}^{2}}{k_{i}^{2}}=\frac{k_{i / /}^{2}+\left(k_{i \perp}^{2}-4 \pi N_{b}\right)}{k_{i}^{2}}=1-\frac{4 \pi N_{b}}{k_{i}^{2}}=1-\frac{\lambda^{2} N_{b}}{\pi}
$$

où $\lambda$ est la longueur d'onde du neutron. Pour la plupart des matériaux, $N_{b}$ étant beaucoup plus petit que 1, une approximation de l'équation (7) conduit au résultat bien connu :

$$
n \approx 1-\frac{\lambda^{2} N_{b}}{2 \pi}
$$


On retrouve encore ici la remarque concernant le changement de longueur d'onde en volume, contraire à celui obtenu avec la diffusion de lumiàre (pour $b$ positif) $: n$ est plus petit que 1 . Le faisceau transmis est donc réfracté à travers le plan du miroir, et à l'angle exact de réflexion totale, le faisceau est réfracté le long de la surface.

Tout le formalisme précédent (à l'exception du traitement des neutrons comme des ondes) peut être obtenu à partir de la physique classique. Cependant, pour pouvoir décrire tous les aspects de la réflectométrie, on va devoir utiliser l'approche de la mécanique quantique.

En séparant les variables $x, y$ et $z$ dans l'équation de Schrödinger, la fonction d'onde associée au neutron près d'une surface s'écrit :

$$
\frac{\partial^{2} \Psi_{z}}{\delta z^{z}}+k_{\perp}^{2}=0 \quad \text { o } \quad k_{\perp}^{2}=\frac{2 m_{n}}{\hbar^{2}}\left(E_{i}-V\right)-k_{/ /}^{2}
$$

Les solutions générales de cette équation au-dessus et en dessous de la surface sont :

$$
\Psi_{z}=e^{i k_{i \perp} z}+r e^{-i k_{i \perp} z} \quad \text { et } \quad Y_{z}=t e^{i k_{t \perp} z}
$$

où $r$ et $t$ sont les amplitudes de diffusion des ondes réfléchie et transmise. Les conditions de continuité de la fonction d'onde à l'interface $(z=0)$ donnent :

$$
1+r=t \quad k_{i \perp}(1-r)=t k_{t \perp}
$$

ce qui conduit directement aux coefficients classiques de Fresnel que l'on trouve en optique :

$$
r=\frac{k_{i \perp}-k_{t \perp}}{k_{i \perp}+k_{t \perp}} \quad \text { et } \quad t=\frac{2 k_{i \perp}}{k_{i \perp}+k_{t \perp}}
$$

En réflectométrie, on mesure la réflectivité en fonction du vecteur de transfert $q$. En combinant les équations (5), (6) et (12), on peut relier la réflectivité $R$ à $q$ et $q_{c}$. Notez que la mesure effectuée est une intensité, donc le carré d'une amplitude.

$$
R=r^{2}=\left[\frac{q-\left(q^{2}-q_{c}^{2}\right)^{1 / 2}}{q+\left(\left(q^{2}-q_{c}^{2}\right)^{1 / 2}\right)}\right]
$$

Lorsque $q>>q c$, l'équation précédente est réduite à :

$$
R \approx \frac{16 \pi^{2}}{q^{4}} N_{b}^{2}
$$

ce qui est la formule de la réflectivité utilisée dans l'approximation de Born [7].

Si on revient à la fonction d'onde à l'intérieur de la surface (10), on trouve en utilisant l'équation (6) que quand $E_{I}<V$ (ou $k_{i \perp}^{2}<4 \pi N_{b}$ ou $q<q-c$ ), une solution réelle est donnée par :

$$
Y_{z}=t e^{i\left(k_{i \perp}^{2}-4 p N_{b}\right)^{1 / 2} z}=t e^{-\frac{1}{2}\left(q_{c}^{2}-q^{2}\right)^{1 / 2} z}
$$

Ce résultat est très important car il montre que lorsque la barrière de potentiel est plus grande que l'énergie de la particule normale à la surface, l'onde peut encore pénétrer sur une profondeur caractéristique de $\left(q_{c}^{2}-q^{2}\right)^{-1 / 2}$. Cette onde évanescente se propage le long de la surface avec un vecteur d'onde $k_{/ /}$et ressort ensuite du volume dans la direction spéculaire (voir Figure 2). Par exemple, pour la valeur de $N_{b}$ de $\operatorname{Si}\left(2.0710^{-6} \AA^{-2}\right)$, la profondeur de pénétration est de l'ordre de $100 \AA$ à $q=0$; elle augmente rapidement à $\infty$ à $q=q_{c}$. Aucune règle de conservation n'est brisée, la réflectivité vaut 1 car cette onde ne représente aucun flux transmis au matériau. Ce résultat explique aussi pourquoi pour 
une couche mince $\left(<100 \AA\right.$ ) d'un matériau tel que $\mathrm{Ni}$, qui a une valeur de $N_{b}$ deux fois plus grande que celle de $\mathrm{Si}$, déposée sur un substrat $\mathrm{Si}$, on retrouve une valeur de $q_{c}$ définie par la valeur de $N_{b}$ du Si et non par celle de Ni. Dans cet exemple, la couche est en fait plus petite que la longueur de pénétration caractéristique de l'onde plane.

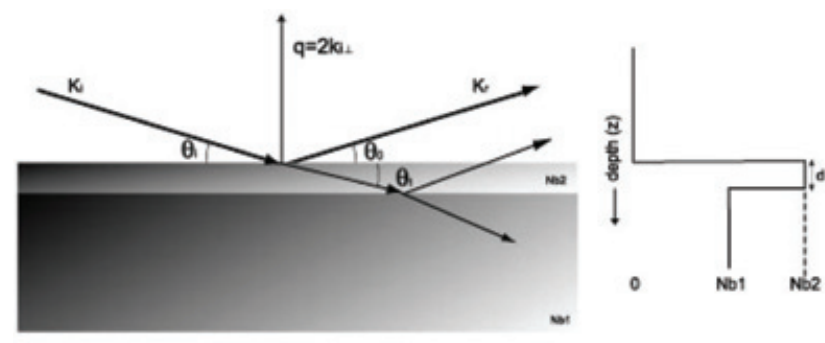

(a)

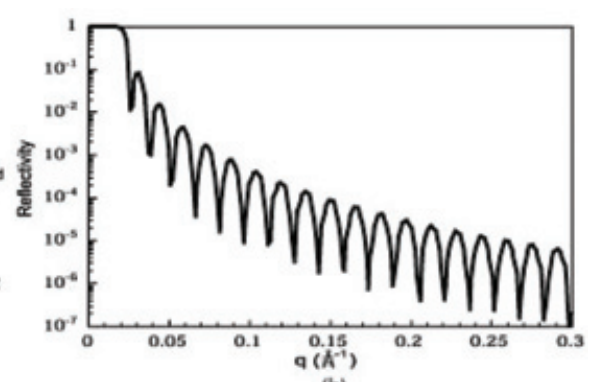

(b)

Figure 2. (a) Voir la légende de la Figure 1(a) avec une couche additionnelle à la surface de densité de longueur de diffusion $\mathrm{Nb}_{2}$. (b) Simulation de la réflectivité spéculaire de neutrons en fonction de q pour une couche de Nickel d'épaisseur de $40 \mathrm{~nm}$ sur un substrat de silicium $\left(\mathrm{Nb}_{2}=9.0410^{-46} \AA^{-2}\right)$.

Pour généraliser le calcul de la réflectivité précédent à un système multicouches, on utilise souvent le formalisme matriciel utilisé en optique [7]. Les transmissions et réflexions entre une couche et la suivante peuvent être obtenues par le produit de matrices correspondant à chaque couche. Le problàme qui consiste à inverser la courbe de réflectivité pour obtenir $N_{b}$ en fonction de la profondeur est compliqué car plusieurs profils peuvent conduire à la même courbe expérimentale. Les diverses techniques d'inversion utilisées peuvent être trouvées dans la revue [8].

\section{Absorption}

En réalité, les matériaux ont aussi une section efficace d'absorption. On en tient compte en ajoutant une partie imaginaire à la longueur de diffusion cohérente :

$$
b_{\text {total }}=b_{\text {cohérent }}+i b_{\text {absorption }}
$$

D'apràs l'équation (10), on voit que les intensités transmise et réfléchie seront exponentiellement réduites par le phénomàne d'absorption. Même dans le régime de la réflexion totale, puisque l'onde évanescente va se propager à la surface du matériau, de l'absorption va se produire, réduisant ainsi l'amplitude de la réflexion totale par rapport à l'unité. On ne doit pas oublier que les matériaux tels que $\mathrm{Cd}$ et $\mathrm{Gd}$, qui sont de très forts absorbants de neutrons, peuvent quand même avoir des réflectivités significatives.

\section{Matériaux magnétiques}

Nous avons vu que l'interaction neutron dépend d'un potentiel moyen relié à une longueur de diffusion cohérente. Si le matériau est magnétique, il faut alors ajouter un potentiel qui décrit l'interaction entre le moment magnétique du neutron et les variations d'induction magnétique créée par les électrons non appariés de l'atome magnétique. Ce potentiel s'écrit

$$
V_{\text {mag }}=-\mu \bullet B(r)
$$

où $\mu$ est le moment magnétique dipolaire du neutron et $B(r)$ les variations du champ magnétique. Dans le cas d'un faisceau incident polarisé, il est possible d'obtenir des neutrons soit tous dans l'état $|+\rangle$ 
(up) (c'est à dire « alignés » avec le champ magnétique) soit tous dans l'état $|-\rangle$ (down) (c'est à dire « alignés » dans le sens opposé au champ), changeant ainsi le signe du potentiel magnétique. Ce potentiel s'exprime aussi par une longueur de diffusion

$$
b_{m}=1.913 \frac{e^{2}}{m_{e}} S
$$

où $e$ et $m_{e}$ sont respectivement la charge et la masse de l'électron et $S$ le spin effectif de l'atome magnétique, perpendiculairement au transfert de moment de l'onde réfléchie.

La longueur totale de diffusion cohérente dépend de la polarisation :

$$
b_{\text {total }}=b_{\text {nucléaire }} \pm b_{\mathrm{m}}
$$

le signe + correspondant à un faisceau incident polarisé dans la même direction que l'aimantation de l'échantillon (- dans le sens opposé).

Après saturation de l'aimantation de l'échantillon, deux mesures seront nécessaires pour déterminer les composantes nucléaire et magnétique de la réflectivité : l'une avec la polarisation parallàle à celle de l'échantillon, $R_{+}$et l'autre avec une polarisation inversée (à l'aide d'un « flipper» [9]), $R_{-}$. Un point important dont il faut se souvenir en réflectométrie de neutrons polarisés (qui sera traitée plus en détails dans ce livre par E. Kentzinger), est qu'il n'y aura réflexion magnétique qu'à unchangement de potentiel et d'après l'équation (17), cela requiert un pas en $B$. Lorsque l'aimantation de l'échantillon est dans le plan de la couche étudiée, les équations de Maxwell prédisent que le pas de champ vaut $\mu_{0} M$ à la surface, où $M$ est la densité d'aimantation de la couche. Il n'y aura réflexion magnétique qu'en présence d'une composante dans le plan de l'aimantation. Les composantes de l'aimantation normales au plan ne réfléchissent pas le faisceau de neutrons polarisés dans le plan, mais elles peuvent produire du « spin flip », c'est à dire que le faisceau de neutrons totalement polarisés aura une certaine fraction de neutrons polarisés dans la direction opposée. Ceci ne peut être mesuré qu'à l'aide d'un second « flipper » au niveau du polariseur ou de l'analyseur. Quatre combinaisons des états de polarisation initiaux et finaux permettent de mesurer quatre réflectivités, $R_{++}, R_{--}, R_{+-}, R_{-+}$. Expérimentalement, un petit champ guide vertical entre le polariseur et l'analyseur permet de conserver la direction de la polarisation selon l'axe vertical. Il faut de plus mesurer les quatre courbes de réflectivité d'un diffuseur non magnétique comme le graphite, afin de déterminer les efficacités des « flipper » et polariseur/analyseur. Une description plus détaillée des effets magnétiques en réflectivité se trouve dans ce livre et dans la référence [9].

\section{Rugosité et interdiffusion}

De nombreuses expériences de réflectivité visent à étudier les interfaces entre plusieurs matériaux. Une interface peut être rugueuse avec des aspérités et des creux sur des grandes échelles de distance, parfois avec une structure de type fractale. Il peut aussi exister une rugosité d'origine magnétique, lorsque l'aimantation ne change pas brutalement à l'interface (voir Figure 3). De même, une inter-diffusion de matériaux va produire des interfaces imparfaites. On se rend compte que pour tous ces types d'interfaces, rugueuse ou diffuse, la réflectivité spéculaire est réduite par un facteur de type Debye-Waller [10]. L'équation (14) s'écrit :

$$
R \approx\left(\frac{16 \pi^{2}}{q^{4}} N_{b}^{2}\right) e^{-q_{z}^{2} \sigma^{2}}
$$

où $\sigma$ caractérise la taille des imperfections de la couche. Que devient l'intensité perdue par ce facteur exponentiel? Dans le cas de l'interface diffuse, cette intensité va dans le faisceau transmis puisqu'il n'y a pas de gradient de potentiel dans une direction autre que celle normale à la surface. Par contre, dans le cas de l'interface rugueuse, l'intensité est perdue par des réflexions locales dans des directions autres 
que la direction spéculaire : c'est la diffusion hors spéculaire. Un paramètre comme la la fonction de corrélation de hauteur peut être obtenu par des mesures de diffusion hors spéculaire [7,11].
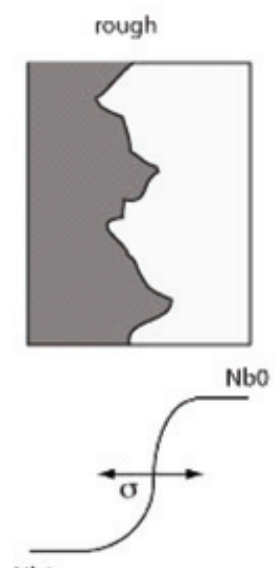

$\mathrm{Nb} 1$
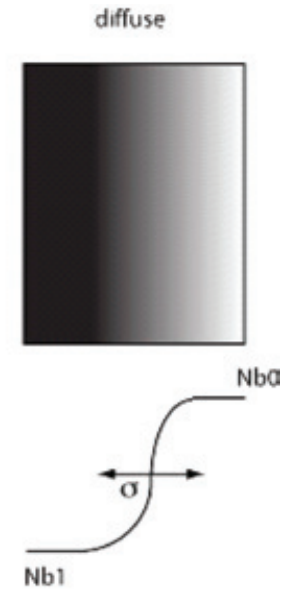

Figure 3. Deux interfaces possibles qui peuvent générer le même profil de réflectivité spéculaire. Dans le cas de l'interface rugueuse, une diffusion hors-spéculaire sera observée.

\section{MESURE DE LA RÉFLECTIVITÉ DE NEUTRONS}

Les composantes principales d'une expérience de réflectivité, soit dans le cas de la lumière, des rayons $x$ ou des neutrons, sont (i) une source de radiation, (ii) un sélecteur de longueur d'onde (monochromateur, choppers), (iii) un systàme de collimation, (iv) l'échantillon et (v) un système de détection.

Les neutrons sont produits soit avec un réacteur nucléaire, où un faisceau continu est produit par fission nucléaire soit avec une source à spallation, où un faisceau pulsé est obtenu quand un paquet de protons ou d'électrons de haute énergie d'un accélérateur sont envoyés dans le noyau d'un élément lourd. Dans les deux cas, les énergies des neutrons sont trop élevées pour des études structurales ou dynamiques : elles sont donc réduites dans des modérateurs où les neutrons sont diffusés en répétition et perdent de l'énergie à chaque collision jusqu'à ce que l'équilibre thermique soit atteint. Les énergies thermiques sont de l'ordre de $k_{B} T$ où $T$ est la température de l'eau du modérateur et $k_{B}$ la constante de Boltzmann.

Le but d'une expérience de réflectivité spéculaire de neutrons est la mesure de la réflectivité en fonction du vecteur d'onde perpendiculaire à la surface réfléchissante, $q$. La mesure peut être faite soit en changeant l'angle d'incidence, $\theta$, soit la longueur d'onde, $\lambda$, des neutrons soit les deux. Dans la plupart des réacteurs nucléaires, les mesures sont normalement faites à une valeur fixée de $\lambda$, en utilisant des neutrons de grande longueur d'onde et des mesures $\theta$ (angle d'incidence) - 2 $\theta$ (angle du détecteur) de 1 intensité. La sélection de la longueur d'onde peut être obtenue par diffusion de Bragg d'un cristal monochromateur ou par sélection de vitesse à travers un disque cylindrique en rotation (sélecteur mécanique). L'intensité du faisceau incidentedoit être aussi mesurée, soit en même temps en utilisant un moniteur, soit avec une mesure complémentaire. La réflectivité de l'échantillon est simplement le rapport de deux intensités pour chaque angle $\theta$, qui est converti en module de vecteur de diffusion $q$ par la loi de Bragg :

$$
q=4 \pi \sin (\vartheta) / \lambda
$$

Avec un échantillon de quelques centimàtres de longueur et un angle $\theta$ initial d'une fraction de degré, le faisceau de neutrons incident doit être bien collimaté pour s'assurer que l'échantillon soit 
sous-illuminé (tout le faisceau incident touche la surface réfléchissante). Les petits échantillons peuvent être sur illuminés, mais les données doivent être corrigées pour tenir compte du fait que le flux de neutrons incident sur échantillon change quand l'angle $\theta$ augmente (voir Figure 4).

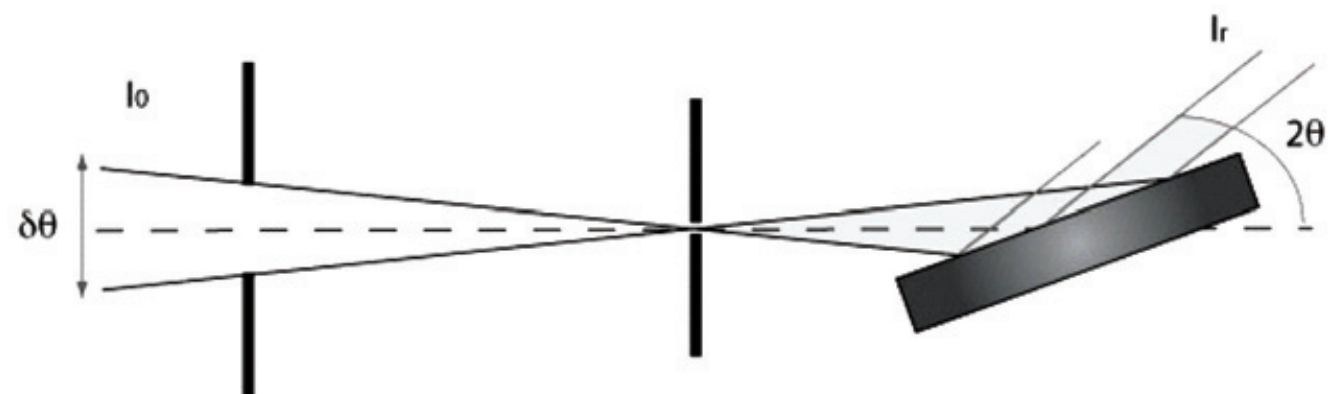

Figure 4. Une configuration de collimation typique dans le plan de réflexion.. Quand l'échantillon est sous-illuminé (bruit de fond minimal), la distance entre la dernière fente du collimateur et l'échantillon devrait être la plus petite possible. En général, la divergence en dehors du plan de réflection est seulement définie par la source.

Le signal de réflectivité décrot très vite quand $q$ augmente (voir (14) ou $R \propto 1 / q^{4}$ ). Néanmoins, pour avoir plus d'intensité (au prix d'une moins bonne résolution), les fentes de collimation peuvent être ouvertes de façon à ce que $\delta \theta / \theta$ soit constant et égal à la variation en longueur d'onde. La courbe de réflectivité finale doit avoir la résolution en $q, \delta q$, ce qui permet de déconvoluer des données brutes. La résolution en $q$ est liée à l'angle $\theta$ et à la longueur d'onde $\lambda$ par la relation suivante :

$$
\left(\frac{\delta q}{q}\right)^{2}=\left(\frac{\delta \theta}{\theta}\right)^{2}+\left(\frac{\delta \lambda}{\lambda}\right)^{2}
$$

Cette méthode présente l'avantage que la longueur d'onde choisie peut correspondre au maximum de la distribution de neutrons de la source et pour une résolution donnée, c'est la méthode la plus efficace pour optimiser le flux de neutrons disponible.

Comme alternative, on peut utiliser la technique de temps de vol («time-of-flight » ou TOF) et c'est cette option qui est le plus souvent utilisée dans les sources à spallation. Dans ce cas, on garde l'angle $\theta$ constant et on utilise toutes les longueurs d'onde présentes dans le faisceau incident. La longueur d'onde, et par conséquent $q$, est mesurée en pulsant le faisceau incident et en mesurant le temps d'arrivée sur le détecteur. La résolution est toujours donnée par l'équation (22) en replaçant $\delta / \lambda$ par $\delta t / t$ ou $\delta$ t est la largeur du canal dans lequel le neutron est détecté et t est le temps de vol, i.e. le temps qu'il met à arriver au détecteur. La gamme de $q$ couverte à un certain angle $\theta$ dépend de la gamme de longueurs d'onde utiles dans le «pulse ». Dans cette gamme, le flux aux longueurs d'onde maximales et minimales peut être plus de deux ordres de grandeur inférieur au flux dans la région du pic. Le vecteur de diffusion $q$ maximum est obtenu pour les longueurs d'onde les plus petites. Pour la même résolution, la méthode TOF est moins efficace que la méthode monochromatique parce qu'elle demande des temps de comptage plus longs pour couvrir la même gamme de q avec la même précision statistique. Par contre, elle est plus favorable pour des mesures de cinétiques. En effet, pour les expériences où la structure à l'interface de l'échantillon change en fonction du temps, seule la méthode TOF peut produire une courbe de réflectivité $R(q)$ unique à un temps donné. Les mesures $\theta-2 \theta$ sont des mesures séquentielles et chaque valeur de $R(q)$ est mesurée à un temps différent. Afin de résoudre le problème du flux réduit inhérent à la méthode TOF, on peut utiliser un système de choppers (disques en rotation avec des fenêtres transparentes aux neutrons) pour changer la résolution en temps et par conséquent la résolution en longueur d'onde [12]. La Figure 5 montre un exemple de réflectomètre qui opàre dans les deux configurations, monochromatique et temps de vol. 


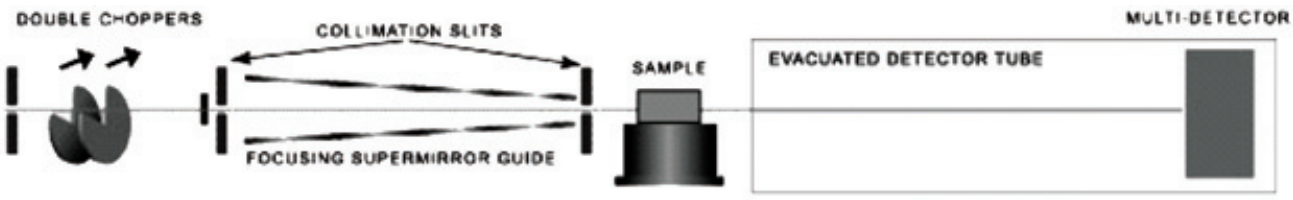

(a)

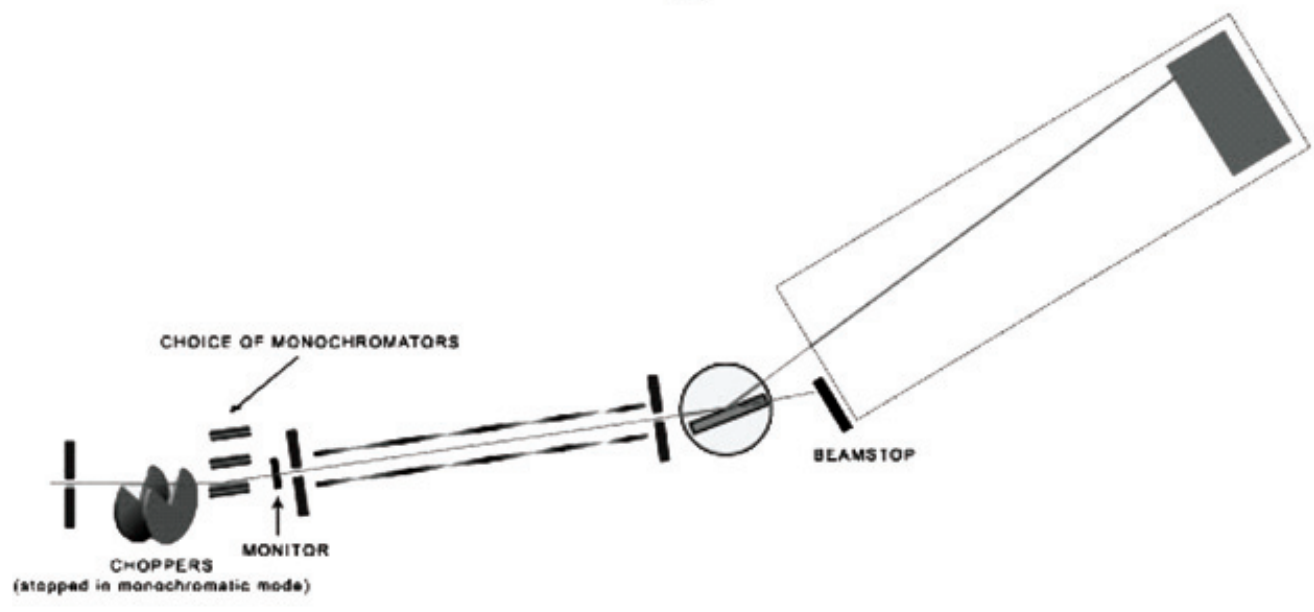

(b)

Figure 5. Le réflectomètre D17 à l'Institut Laue-Langevin [12]. (a) Vue de côté de l'instrument dans le mode TOF. Le monochromateur est enlevé et un système de choppers définit la résolution en temps (1-20\% $\delta \mathrm{t} / \mathrm{t})$. Entre les fentes de collimation, il y a un guide focalisant dans la direction verticale qui produit une augmentation du flux à la position de l'échantillon au prix d'une plus grande divergence verticale du faisceau. Les fentes définissent la taille du faisceau dans la direction horizontale. (b) Vue verticale de l'instrument en mode monochromatique. Le bras de collimation est tourné d'environ 4ř pour permettre au faisceau réfléchi par le monochromateur multicouches de passer à travers l'échantillon.

En augmentant $q$, le niveau du bruit de fond augmente en raison du fait que tous les neutrons ne sont pas réfléchis de façon spéculaire. Pour les grandes valeurs de $q$, la réflectivité est trés petite et la plupart du faisceau traverse le substrat (par exemple l'eau) où il est diffusé, soit de façon incohérente, soit par diffraction multiple. Une partie de ce faisceau diffusé arrive sur le détecteur avec la réflexion spéculaire et contribue au bruit de fond : il doit être soustrait du profil de réflectivité.

Il y a d'autres sources de bruits de fond en relation avec l'environnement de l'échantillon et des composantes du réflectomètre. Dans la plupart des cas, le signal de diffusion incohérente de l'échantillon même représente la source majeure de bruit. La diffusion incohérente comprend un nombre de phénomènes de diffusion qui sont complètement casuels et proviennent du fait que tous les noyaux d'un élément donné ne sont pas identiques à cause de l'existence de différents isotopes ou de différents états de spin. Heureusement, cette diffusion est isotrope.

Autour du faisceau spéculaire réfléchi, il $y$ a alors un signal de bruit de fond qui doit être soustrait avant de faire le rapport du faisceau réfléchi avec le faisceau incident. Ce bruit de fond peut être tràs important pour des expériences qui impliquent des interfaces avec l'eau et la réflectivité est limitée dans ces cas à $\sim 10^{-7}$. En utilisant un détecteur bidimensionnel, une grande gamme d'angles $2 \theta$ est interceptée, incluant bruit de fond et diffraction hors-spéculaire. Pour les instruments équipés seulement d'un détecteur ponctuel, la mesure du bruit de fond requiert une mesure à part du signal hors-spéculaire. 
Dans les deux cas, il faut sélectionner avec attention la région en $2 \theta$ utilisée pour déterminer ce bruit de fond et s'assurer qu'elle ne contient pas d'intensité diffractée intéressant la structure interfaciale de l'échantillon.

Les expériences utilisant les neutrons polarisés (Polarized Neutron Reflectometry, PNR), sont compliquées par le fait que normalement les polariseurs et flippers ne sont pas parfaits. En l'absence d'échantillon et avec le faisceau incident arrivant directement sur l'analyseur, on n'attend pas d'intensité sur le détecteur dans les conditions -+ ou +- pour un systàme parfait. Une mesure de l'efficacité de chaque renverseur («flipper »)et polariseur est le rapport de renversement (rapport de « flipper») :

$$
F_{1}=I_{++} / I_{-+} \quad \text { and } \quad F_{2}=I_{++} / I_{+-}
$$

où $F_{1}$ et $F_{2}$ sont les rapports de renversement pour le premier et le deuxième renverseur. Les intensités sont mesurées, soit avec le faisceau direct pour un détecteur ponctuel, soit avec un diffuseur non magnétique pour un détecteur bi-dimensionnel. Les intensités mesurées correspondent aux états des deux renverseurs comme indiqués dans la Figure 3. Des rapports de renversement acceptables sont de l'ordre de 40. Ils doivent être considérés quand les composantes magnétiques et nucléaires de la réflectivité sont extraites d'une courbe expérimentale [9]. Des exemples seront donnés dans les cours de M. Viret et F. Ott ainsi que dans celui d'E. Kentzinger (ce livre).

\section{ANALYSE DES DONNÉES}

La méthode d'analyse très souvent utilisée pour les données de réflectivité spéculaire implique la construction d'un modèle de l'interface constitué par une série de couches parallèles de matériel homogène. Chaque couche est caractérisée par une densité de longueur de diffusion, $\rho$, et une épaisseur, $d$, qui sont utilisées pour calculer un profil de réflectivité modèle en utilisant la méthode de la matrice optique. La rugosité à l'interface entre deux couches consécutives, peut être aussi incluse dans le modèle par la méthode d'Abeles [13]. Le profil calculé est comparé avec le profil mesuré et la qualité du fit vérifiée, soit en utilisant le $\chi^{2}$, soit la méthode de la moyenne quadratique. Les deux extrêmes de l'interface sont considérés avoir une épaisseur infinie et une densité de longueur de diffusion fixée. À l'interface, il $y$ a, soit une simple couche uniforme, soit une structure plus compliquée. En changeant $\rho$ ou $d$ pour chaque couche, le profil calculé peut être comparé avec le profil mesuré. jusqu'à la détermination de l'affinement optimal pour les données. Chaque profil peut être décrit par plus d'un modèle. En matière molle, l'utilisation de différents contrastes isotopiques, en plus des contraintes physiques et chimiques du système, peut en général assurer qu'un modèle non ambigu de l'interface soit déterminé. La variation de contraste repose sur le fait que les noyaux diffusent les neutrons avec une amplitude différente et parfois, comme dans le cas des protons et deutérons, avec une phase opposée. En utilisant un mélange de matériaux hydrogénés et deutériés, le profil de réflectivité d'un systàme peut être changé en gardant la même structure à l'interface. Il est aussi possible en ajustant le rapport H/D de préparer des solvants ayant la même densité de longueur de diffusion du substrat à travers lequel les neutrons passent avant d'arriver à l'interface, i.e. le solide pour une interface solide/liquide ou l'air pour les interfaces solide/air et liquide/air. Le contraste entre le solide (ou air) et le solvant devient nul et le profil de réflectivité dépend seulement de la région interfaciale. A partir de la valeur de la densité de longueur de diffusion à l'interface, on obtient des informations sur sa composition.

L'approximation cinématique [14] donne une description plus directe de la structure de monocouches solubles de tensioactifs à 1 'interface air/liquide. Récemment, Majkrzak et al. [15] ont exploité une méthode sensible à la phase (phase-sensitive) pour révéler des profils de composition en fonction de la profondeur. Avec cette nouvelle méthode, le profil de densité de longueur de diffusion pour des membranes biomimétiques est obtenu à partir d'une inversion sans avoir besoin d'affiner ou ajouter des paramàtres. Les autres approches qui n'utilisent pas des modèles se servent de la méthode du maximum d'entropie [16] ou de B-splines [17]. 


\section{RÉFLECTIVITÉ DE NEUTRONS ET MATIÈRE MOLLE}

Les applications en matière molle sont souvent les plus courantes pour la technique de réflectivité de neutrons. Les systèmes de matière molle sont des systèmes complexes comme les polymères et élastomères, les cristaux liquides, les mousses, les tensioactifs, les lipides et les protéines. Ces systèmes sont importants autant dans le domaine de la biologie que pour la synthèse de matériaux avancés, la technologie de la nutrition, la récupération du pétrole et dans les nouvelles biotechnologies comme l'envoi ciblé de médicaments dans les cellules.

Comme déjà évoqué précédemment, la réflectivité spéculaire des neutrons permet d'obtenir la distribution des indices de réfraction ou densité de longueur de diffusion perpendiculaire à une surface ou une interface, et donc en relation directe avec la composition ou le profil de concentration dans la région de l'interface. La géométrie en incidence rasante et la gamme de longueurs d'onde des neutrons froids permettent de sonder les échelles intéressantes en matière molle qui vont de $\sim 1 \mathrm{~nm}$ jusqu' à $400 \mathrm{~nm}$. Les pouvoirs de diffusion de l'hydrogàne et du deutérium sont tràs différents et permettent la manipulation de la distribution d'indices de réfraction avec la substitution isotopique (échange H/D), sans modifier notablement la structure des systèmes. La possibilité de manipuler le contraste est une caractéristique puissante et très utilisée. Il est ainsi possible de mettre en évidence des parties de l'interface, d'étudier l'interface d'une bicouche de polymères, ou de sélectionner des composants dans des mélanges complexes adsorbés aux interfaces. Les neutrons froids sont aussi très pénétrants et permettent l'étude d'interfaces cachées. Les études ne sont pourtant pas limitées aux interfaces air-liquide et air-solide, mais peuvent être menées aussi aux interfaces solide-solide, solide-liquide et liquide-liquide. Dernièrement, le rayonnement synchrotron s'est imposé comme une technique complémentaire à celui des neutrons dans l'étude de ces interfaces $[18,19]$. La limitation de ce type de rayonnement est liée à l'endommagement des systàmes mous, ce qui rend son utilisation en matière molle plus délicate par rapport à la réflectivité de neutrons qui elle ne dégrade pas les échantillons étudiés.

Les nouvelles applications de la technique impliquent les études d'interfaces complexes comme celles des membranes biologiques, l'électrochimie « in-situ », l'adsorption aux interfaces liquide-solide et liquide-liquide, et des environnements complexes comme des surfaces sous cisaillement ou confinées. Dans l'étude de l'adsorption des polymères et de tensioactifs, la tendance est d'étudier le développement de nano-structures auto-assemblées qui se forment.

Enfin, des mesures d'électrochimie « in situ » sont décrites par exemple dans la référence [20] ou la mesure de la pénétration de solvants dans des films électro-polymérisés a été possible. Quelques mesures de fluides complexes confinés entre des surfaces de quartz et de saphir séparées par $\sim 100 \mathrm{~nm}$ sont décrites dans la référence [21]. L'effet de l'épaisseur de couches de polymères à bloc immiscibles sur le degré d'inter-diffusion à l'interface est décrit dans la référence [22].

L'étude de l'interface liquide-liquide mérite une mention particulière vu son importance en technologie et la difficulté de la sonder avec des techniques traditionnelles. Bowers et al. [23] ont établi une méthodologie de préparation qui consiste à créer une couche très fine d'huile entre un substrat de silice rendu hydrophobe et une solution aqueuse. Les neutrons traversent la silice pour atteindre l'interface.

La réflectivité de neutrons reste pourtant une technique à faible résolution. Son exploitation optimale dépend de la possibilité de mener des études complémentaires sur les systèmes étudiés.

Les manuscrits qui suivront cette introduction à la technique de réflectométrie vont fournir des nombreux exemples d'applications. Pour plus de détails, le lecteur peut aussi consulter des revues récentes sur les études par réflectivité de neutrons en matière molle [24,25] ou visant particulièrement le domaine des interfaces de polymères [26], la biologie [27,28], les tensioactifs et mélanges polymères-tensioactifs $[29,30]$.

\section{Remerciement}

Un grand merci à Annie Brûlet pour son aide dans la rédaction de ce texte en français. 


\section{Références}

[1] Fermi, E. and Zinn, W. H., Phys. Rev. 70, 103 (1946).

[2] Penfold, J. and Thomas, R. K., J. Phys. Condens. Matter 2, 1369 (1990).

[3] Felcher, G. P., Phys. Rev. B 24, 1995 (1981).

[4] Sinha, S. K., Sirota, E. B., Garoff, S. and Stanley, H. B., Phys. Rev. B 38, 2297 (1998).

[5] Sears, V. F., "Neutron Optics", Oxford Press, Oxford (1989).

[6] Lekner, J., in “Theory of Reflection” Martinus Nijhoff Dordrecht (1987).

[7] Born, M. and Wolfe, E., in "Principles of Optics" Pergamon Press Eds. Oxford (1989).

[8] Zhou, X. L. and Chen, S. H., Phys. Rep. 257, 223 (1995).

[9] Williams, W., in "Polarized Neutrons", Oxford Univ. Press, Oxford (1989).

[10] Névot, L. and Croce, P., Rev. de Phys. Appl. 15, 761 (1990).

[11] Daillant, J. and Gibaud, A., in "X-ray and Neutron Reflectivity : Principles and Applications" Springer Eds (1999).

[12] Cubitt, R. and Fragneto, G., Appl. Phys. A 74, pp. S329-S331.(2002).

[13] Heavens, O. S., in "Optical Properties of Thin Films", Butterworths Eds. London (1955).

[14] Crowley, T. L., Physica A 195, 354(1993).

[15] Majkrzak, C. F., Berk, N. F., Krueger, S., Dura, J. A., Tarek, M., Tobias, D., Silin, V., Meuse, C. W., Woodward, J. and Plant, A., Biophys. J. 79, 3330 (2000).

[16] Silvia, D. S., Hamilton, W. A. and Smith, G. S., Physica B 173, 121 (1991).

[17] Berk, N. F. and Majkrzak, C. F., Phys. Rev. B 51, 11, 296 (1991).

[18] Daillant, J., Mora, S., Fradin, C., Alba, M., Braslau, A. and Luzet, D., Appl. Surf. Sci., 182, 3-44, 223-230.

[19] Daillant, J., Bellet-Amalric, E., Braslau, A., Charitat, T., Fragneto, G., Graner, F., Mora, S., Rieutord, F. and Stidder, B., Proc. Nat. Acad. Sci. 102 (33), 11639 (2005).

[20] Cooper, J. M., Cubitt, R., Dalglesh, R. M., et al., J. Am. Chem. Soc. 126 (47), 15362-15363 (2004).

[21] Kuhl, T. L., Smith, G. S., Isrealachivili, J. N., Majewski, J., Hamilton, W., Rev. Sci. Inst. 72, 1715 (2001).

[22] Carelli, C., Jones, R. A. L., Young, R. N., Cubitt, R., Dalgliesh, R., Schmid, F. and Sferrazza, M., Phys. Rev. E 72, 031807 (2005).

[23] Bowers, J., Zarbakhsh, A., Webster, J. R. P., Hutchings, L.R., Richards, R.W., Langmuir 17, 140 (2001).

[24] Penfold, J., et al., J. Chem. Soc. Faraday Trans. 93, 22, 3899 (1997).

[25] Penfold, J., Cur. Opin. Coll. Int. Sci. 7, Issues 1-2, 139-147 (2002).

[26] Sferrazza, M., Jones, R. A. L, Penfold, J., Bucknall, D. G., Webster, J. R. P., J. Mat. Chem. 10, 127 (2002).

[27] Krueger, S., Cur. Opin. Coll. Int. Sci. 6, 111 (2001). Fragneto-Cusani, G., J. Phys. Cond. Mat. 13, 4973 (2001).

[28] Lu, J. R., Thomas, R. K., Penfold, J., Adv. Cond. Int. Sci. 84,143 (2000).

[29] Thomas, R. K., Ann. Rev. Phys. Chem. 55, 391-426 (2004). 